\title{
Cutaneous Histoplasmosis and Role of Direct Microscopy-A Case Report
}

Malhotra $\mathbf{S}^{*}$, Dhundial R, Kaur NJK, Kaushal M and Duggal N

Department of Microbiology and Pathology, PGIMER associated Dr Ram Manohar Lohia Hospital, New Delhi

*Corresponding author: $\mathrm{Dr}$ Shalini Malhotra, Department of Microbiology, PGIMER associated Dr Ram Manohar Lohia Hospital, New Delhi, India, Tel: +91-7428177617; E-mail: drshalinimalhotra@yahoo.com

Received date: February 21, 2016; Accepted date: March 15, 2017; Published date: March 25, 2017

Copyright: (c) 2017 Malhotra S, et al. This is an open-access article distributed under the terms of the Creative Commons Attribution License, which permits unrestricted use, distribution, and reproduction in any medium, provided the original author and source are credited.

\begin{abstract}
Histoplasmosis is an important systemic fungal infection in endemic areas. It has been reported from various states of India, with particular endemicity in north-eastern regions. Most infections are asymptomatic or self-limited, some individuals develop acute pulmonary infections or severe and progressive disseminated infection. Primary infection occurs in lungs and can also involve skin, lymph nodes, GIT, CNS, adrenals, liver and spleen. The particular interest of this paper is to present a case of disseminated histoplasmosis in diabetic patient and to demonstrate the importance of direct $\mathrm{KOH}$ mount preparation for diagnosis of such cases.
\end{abstract}

Keywords: Cutaneous; Disseminated; Histoplasmosis; $\mathrm{KOH}$

\section{Introduction}

Disseminated histoplasmosis is caused by Histoplasma capsulatum which is a dimorphic fungus found in soil infected with bird and bat excreta [1]. The predisposing factors for histoplasmosis are immunosuppressed patients, acquired immunodeficiency disease syndrome, malignancy, solid organ or bone marrow transplant, and high dose or prolonged steroid therapy [2,3]. Most of the time infection remains asymptomatic or self-limited except in $5-10 \%$ cases which may develop progressive disseminated disease [4]. Disseminated and extrapulmonary histoplasmosis is commonly seen among immunocompromised individuals especially from endemic region and clinically presents with non-specific symptoms like pyrexia, anorexia, nausea, vomiting, weightloss, and fatigue. These nonspecific symptoms can resemble other chronic infections including tuberculosis and malignancies and can lead to diagnostic dilemma [5,6]. The organs commonly involved in disseminated histoplasmosis are liver, spleen, adrenal gland, bone marrow and lymph node [2,7]. The skin involvement, commonly seen in HIV positive cases, is rare in disseminated disease and occurs in only $6 \%$ of patients in the form of hyperpigmented, erythematous nodule, papule, or ulcerative lesions [8]. Here we report a case of disseminated histoplasmosis with cutaneous lesions in a 45 year old male diabetic patient from New Delhi, India. This case report highlights the presenting features and role of $\mathrm{KOH}$ preparation in the diagnosis.

\section{Case Report}

A 46-year-old male, vegetable vendor, diabetic, resident of Delhi, North India, presented with complaints of multiple plaques and nodules on his face, neck, upper arms and trunk since 1 month. He had sudden onset fever 3 months back. He showed to a private hospital where he was diagnosed as malaria and was put on chloroquine and other medications. However no signs of improvement were found and patient was referred to another hospital where he was put on broad spectrum antibiotics and oral antifungal along with topical ointments. One month following this, he developed skin lesions over the nape of his neck. Fever was persisting throughout the time and patient also developed mild cough. There were no other systemic complaints. Cutaneous examination showed multiple erythematous, discrete, nontender, firm papules, over the face, neck, trunk, upper limbs, lower limbs and back. On physical examination, bilaterally enlarged, matted and non-tender inguinal lymph nodes were seen. The presentation of patient created a clinical dilemma between infective and non-infective causes and a probability of Non-Hodgkin's Lymphoma was also kept in mind. Therefore all relevant laboratory investigations like Haemogram, liver and kidney blood chemistries and electrolytes were performed and found to be within normal limits. Chest X-ray showed pneumonitic infiltrations involving right upper zone. HIV and VDRL test were negative. The peripheral blood CD4 count was 285 cells $/ \mathrm{mm}^{3}$ (using FACS caliber).

\section{Methodology}

Thereafter a skin biopsy was performed and sent to microbiology lab. The biopsy was homogenized using tissue homogenizer and then $10-20 \% \mathrm{KOH}$ was added and left overnight. Next day the sample was centrifuged at $2000 \mathrm{rpm}$ for 10 minutes and then a direct $\mathrm{KOH}$ mount was prepared on a slide which showed intracellular budding yeast cells with clear space around them based on which the fungus provisionally was identified as Histoplasma capsulatum (Figure 1). The biopsy was also processed for $\mathrm{H} \& \mathrm{E}$ staining whereby the specimen was deparaffinize and placed in xylene. Hydration was performed by passing through decreasing concentration of alcohol baths and water. $(100 \%, 90 \%, 80 \%, 70 \%)$ followed by staining in hematoxylin for 3-5 minutes. Then washing was performed in running tap water until sections "blue" for 5 minutes or less followed by $1 \%$ acid alcohol (1\% $\mathrm{HCl}$ in $70 \%$ alcohol) for 5 minutes. Washing in running tap water was performed until the sections were again blue by dipping in an alkaline solution (eg. ammonia water) followed by tap water wash. This was then stained in $1 \%$ Eosin Y for 10 minutes and was washed in tap water for 1-5 minutes followed by dehydration in increasing concentration of alcohols. FNAC of inguinal lymph nodes was also performed and sent simultaneously to pathology lab where on giemsa stain showed numerous histiocytes studded with yeast form along with extracellular yeast cells seen (Figure 2). H\&E stain confirmed the presence of fungal yeast cells (Figure 3). Based on clinical, 
microbiological and pathological findings case was diagnosed as a case of disseminated histoplasmosis with primary lung involvement.

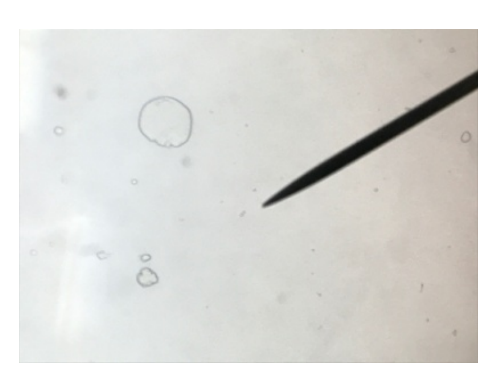

Figure 1: $\mathrm{KOH}$ mount of skin biopsy showing intracellular yeast form of Histoplasma spp. The pointer shows budding yeast cell form inside the macrophage giving the false impression of capsule.

\section{Discussion}

In India, histoplasmosis is not considered endemic, but areas of high prevalence are along the north-eastern states, namely West Bengal and Uttar Pradesh $[9,10]$. First case of histoplasmosis from India was described by Panja and Sen [11]. Since then it has been reported from various states (eg. Chandigarh, Bihar, Rajasthan, Madhya Pradesh, Gujarat, Chattisgarh and Kerala) as isolated case reports as well as case series among both immunocompromised and healthy patients. The patient in this study was a 46-year-old- diabetic male and a resident of Delhi in North India. Delhi has previously not been considered endemic but some cases have been reported recently [12-14].

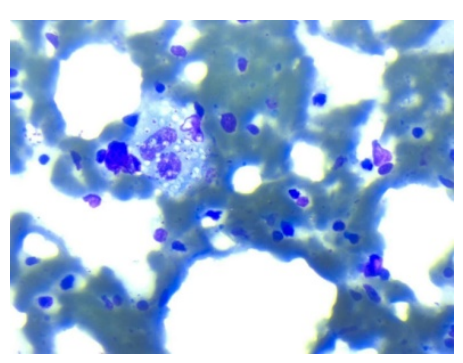

Figure 2: Giemsa stained mount of skin biopsy showing intracellular yeast form of Histoplasma spp.

Disseminated histoplasmosis develops mostly in patients who are immunocompromised. Diabetes and AIDS are common co-morbid conditions seen with disseminated histoplasmosis in some Indian studies $[15,16]$. In the present case, diabetes and low CD4 counts $(285$ cells $/ \mathrm{mm}^{3}$ ) were accountable for the immunocompromising condition of the patient. The risk factors for acquiring the infection include, occupations involving disruption of soil rich in bird and bat guano like, agriculture, outdoor construction and rehabilitation of buildings inhabited by birds [17]. The present patient was a vegetable vendor by occupation with exposure to farming in autumn season. He could have been exposed to contaminated soil during the course of this work.

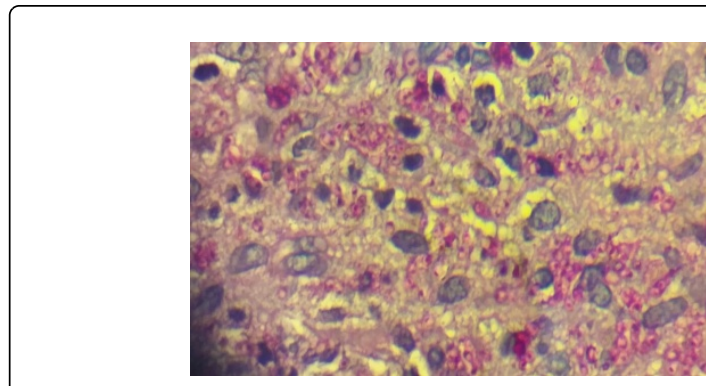

Figure 3: H\&E stained mount of Lymph node biopsy showing intracellular yeast form of Histoplasma spp.

Our patient had enlarged liver, spleen and bilateral inguinal lymph nodes along with erythematous papules on face, trunk, back and on limbs. Hepatosplenomegaly with cutaneous lesions were a significant mode of presentation in this case as well as in other reports by many Indian authors. [11,18-20]

The case was diagnosed as disseminated histoplasmosis on the basis of clinical presentation and direct microscopy and confirmation by histopathology. Although culture was negative, $\mathrm{KOH}$ mount features were characteristic for diagnosis (Figure 2). Culture could have been negative due to some antifungal treatment in past as patient had gone to a nursing home before presenting to our institute. Hence, this case report emphasizes the role of direct $\mathrm{KOH}$ mount preparation for the diagnosis of histoplasmosis. In Indian literature, very few reports demonstrated the diagnosis of disseminated histoplasmosis solely on $\mathrm{KOH}$ microscopy results. To best of our knowledge there is only one case of disseminated histoplasmosis with cutaneous manifestation that has been reported from India in which microbiological diagnosis was made solely on the basis of direct $\mathrm{KOH}$ mount preparation [21].

The early clinical manifestations of disseminated histoplasmosis are nonspecific, often lead to diagnostic difficulty, and it is misdiagnosed as tuberculosis or malignancy [22]. Therefore clinical awareness and good diagnostic laboratory facilities are all necessary for improved understanding of the epidemiology of histoplasmosis as well as to differentiate disseminated disease from more common diseases in our country like tuberculosis, lymphoma or metastatic malignancy.

\section{References}

1. Gupta P, Bhardwaj M (2016) Cytodiagnosis of disseminated histoplasmosis in an immunocompetent individual with molluscum contagiosum-like skin lesions and lymphadenopathy. J Cytol 33: 163-165.

2. Goodwin RA, Shapiro JL, Thurman GH, Thurman SS, Prez Des RM (1980) Disseminated histoplasmosis: clinical and pathologic correlations. Medicine (Baltimore) 59: 1-33.

3. Gupta N, Arora SK, Rajwanshi A, Nijhawan R, Srinivasan R (2010) Histoplasmosis: Cytodiagnosis and review of literature with special emphasis on differential diagnosis on cytomorphology. Cytopathology 21: 240-244.

4. Kauffman CA (2007) Histoplasmosis: a clinical and laboratory update. Clinical Microbiology Reviews 20: 115-132.

5. Wheat LJ (2006) Histoplasmosis: a review for clinicians from nonendemic areas. Mycoses 49: 274-282.

6. Grover SB, Midha N, Gupta M, Sharma U, Talib VH (2005) Imaging spectrum in disseminated histoplasmosis: case report and brief review. Australasian Radiology 49: 175-178. 
Citation: Malhotra S, Dhundial R, Kaur NJK, Kaushal M and Duggal N (2017) Cutaneous Histoplasmosis and Role of Direct Microscopy-A Case Report. Clin Microbiol 6: 276. doi:10.4172/2327-5073.1000276

Page 3 of 3

7. Rog CJ, Rosen DG, Gannon FH (2016) Bilateral adrenal histoplasmosis in an immunocompetent man from Texas. Medical Mycology Case Reports 14: 4-7.

8. Hinshaw M, Longley BJ (2010) Fungal diseases. In: Elder DE, Elenitsas R, Johnson BL Jr, Murphy GF, Xu X, editors. Lever's Histopathology of the Skin. 10th ed. New Delhi: Lippincott Williams \& Wilkins 611.

9. Randhawa HS, Khan ZU (1994) Histoplasmosis in India: current status. Indian J Chest Allied Sci 36: 193-213.

10. Padhye AA, Pathak AA, Katkar VJ, Hazare VK, Kaufman L (1994) Oral histoplasmosis in India: a case report and an overview of cases reported during 1968-92. J Med Vet Mycol 32 : 93-103.

11. Panja G, Sen S (1954) A unique case of histoplasmosis. J Ind Med Assoc 23: $257-258$

12. Ahuja A, Mathur SR, Iyer VK, Sharma SK, Kumar N, et al. (2012) Histoplasmosis presenting as bilateral adrenal masses: Cytomorphological diagnosis of three cases. Diagn Cytopathol 40: 729-731.

13. Dhawan J, Verma P, Sharma A, Ramam M, Kabra SK, et al. (2010) Disseminated cutaneous histoplasmosis in an immunocompetent child relapsed with itraconazole, successfully treated with voriconazole. Pediatr Dermatol 27: 549-551.

14. Bhattacharya JB, Rani P, Aggarwal R, Kaushal S (2017) Primary Cutaneous Histoplasmosis Masquerading as Lepromatous Leprosy. Journal of Clinical and Diagnostic Research 11: 1-2.
15. Subramanian S, Abraham OC, Rupali P, Zachariah A, Mathews MS, et al. (2005) Disseminated Histoplasmosis. J Assoc Physicians India 53: 185-189.

16. Gopalakrishnan R, Nambi PS, Ramasubramanian V, Abdul Ghafur K, Parameswaran A (2012) Histoplasmosis in India: truly uncommon or uncommonly recognised? J Assoc Physicians India 60: 25-28.

17. Hajjeh RA, Pappas PG, Henderson H, Lancaster D, Bamberger DM, et al. (2001) Multicenter case control study of risk factors for histoplasmosis in Human Immunodeficiency virus- infected persons. Clin Infect Dis 32: 1215-1220.

18. Bahri HC, Sagreiya K, Bassi NK, Rohatgi MS (1972) Histoplasmosis of the larynx and tongue. J Laryngol \& Otol 86: 285-291.

19. Mukherjee AM, Khan KP, Sanyal M, Basu N (1971) Histoplasmosis in India with report of two cases. J Ind Med Assoc, 56: 121-125.

20. Radhakrishnan S, Vanchilingam S, Subramaniam S, Venkatraman ITV (1982) Acute disseminated histoplasmosis with unusual features-an autopsy report. J Assoc Phys India 30: 45-48.

21. Gosavi M, Pande S, Mahajan H, Oke M (2015) Primary cutaneous histoplasmosis: a case report with review of literature. IJMPS 5: 5-7.

22. Sharma S, Kumari N, Ghosh P, Aggarwal A (2005) Disseminated histoplasmosis in an immunocompetent individual-A case report. Indian J Pathol Microbiol 48: 204-246. 